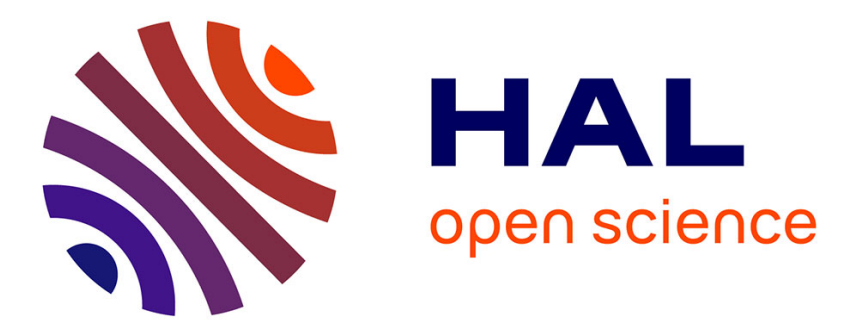

\title{
Finite element modeling for temperature stabilization of gated Hall sensors
}

Benoit Jouault, L. Bouguen, Sylvie Contreras, A. Kerlain, Vincent Mosser

\section{To cite this version:}

Benoit Jouault, L. Bouguen, Sylvie Contreras, A. Kerlain, Vincent Mosser. Finite element modeling for temperature stabilization of gated Hall sensors. Journal of Applied Physics, 2008, 104 (5), pp.053705. 10.1063/1.2968436 . hal-00388502

\section{HAL Id: hal-00388502 https://hal.science/hal-00388502}

Submitted on 26 May 2009

HAL is a multi-disciplinary open access archive for the deposit and dissemination of scientific research documents, whether they are published or not. The documents may come from teaching and research institutions in France or abroad, or from public or private research centers.
L'archive ouverte pluridisciplinaire HAL, est destinée au dépôt et à la diffusion de documents scientifiques de niveau recherche, publiés ou non, émanant des établissements d'enseignement et de recherche français ou étrangers, des laboratoires publics ou privés. 


\title{
Finite Element Modeling for Temperature Stabilization of Gated Hall Sensors
}

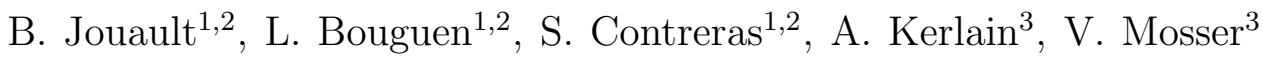 \\ ${ }^{1}$ Université Montpellier 2, Groupe d'Étude des Semiconducteurs, \\ cc074, pl. Eugène Bataillon, 34095 Montpellier cedex 5, France \\ ${ }^{2}$ CNRS, UMR 5650, cc074, pl. Eugène Bataillon, \\ 34095 Montpellier cedex 5, France and \\ ${ }^{3}$ ITRON SAS, 76 avenue Pierre Brossolette, 92240 Malakoff, France
}

\begin{abstract}
Using finite element analysis, we have calculated the Hall voltage of gated Hall sensors in the temperature range $\left[-55^{\circ} \mathrm{C}, 125^{\circ} \mathrm{C}\right]$. We investigated how both the sensor shape and the external connections influence the Hall voltage and its thermal drift. The numerical results are in excellent agreement with the experimental measurements. By contrast, we checked that simplified analytical methods lead to a large numerical error which is not acceptable in these sensors devoted to metrological applications. In particular, it is found that the thermal drift of the Hall voltage can be canceled for a current of the order of $300 \mu \mathrm{A}$, a much higher value than that predicted by the corresponding analytical calculations.
\end{abstract}

PACS numbers: 72.20.My, 85.30.De, 72.80.Ey 


\section{INTRODUCTION}

Hall effect sensors are widely used for industrial applications and fundamental research ${ }^{1}$. They cover a large range of topics, from the study of carrier transport phenomena in solids to the detection of a local magnetic field. An important factor of merit in Hall sensors is the Hall sensitivity $K_{H}=V_{H} /(I B)$, where $V_{H}$ is the Hall voltage, $I$ the injected current and $B$ the magnetic field perpendicular to the plane defined by the electrodes. For a given application, the required amplitude and stability of this Hall sensitivity usually determine the choice of specific materials and designs. Very often, sensors are made of GaAs, because: i) this material can be designed with low carrier concentrations, which is a prerequisite to obtain a high Hall voltage $V_{H}$; ii) it offers the possibility of using mature micro-electronic technologies and iii) it has a large band-gap, which minimizes the temperature drift of $K_{H}$. For metrological applications, two-dimensional electron gas (2DEG) Hall sensors offer a greater precision, a good temperature stability ${ }^{2}$, with a noise maintained at a low level ${ }^{3,4}$. Fig. 1 shows a typical pseudomorphic GaAs heterostructure designed for Hall sensors, in which a Si doping layer induces a 2DEG of concentration $n_{0}$ in the Quantum Well (QW). In this case, $K_{H}$ is given by:

$$
K_{H}=G \frac{r_{H}}{n_{0} e},
$$

where $-e$ is the electron charge, $r_{H}$ is a material parameter which is usually called the Hall coefficient and $G$ is a geometric factor which depends on the device shape. If the 2DEG is degenerate, which is usually the case even at room temperature, then $r_{H} \sim 1$. The $G$ factor can be chosen very close to unity by designing a homogeneous Greek cross whose length is much greater than the width. If all these conditions are met, then $K_{H}$ is a direct measurement of the carrier density $n_{0}$. However, at room temperature, even these sensors present a residual thermal drift of $K_{H}$. The reason is as follows: the surface states are in thermal equilibrium with the 2DEG because of thermionic processes. These surface states are well known features of the GaAs; they correspond to dangling bonds at the GaAs surface. The population of the surface states is strongly $T$-dependent, hence the Fermi energy varies and $n_{0}$ and $K_{H}$ are also $T$ dependent, even if great care was taken to increase the distance between the surface and the 2DEG. As a last resort, the $T$-dependence has to be compensated by additional electronics.

In this paper, we present the method we developed to stabilize 2DEG Hall sensors over 
the temperature range $\left[-55^{\circ} \mathrm{C},+125^{\circ} \mathrm{C}\right]$, which corresponds to the military and extended industrial specifications. The dramatic reduction of the temperature sensitivity is reached by optimizing the geometric contribution $G$ and by controlling the population of surface states by an additional gate. In this approach, $G$ is not equal to one and therefore it has to be calculated numerically. It has been demonstrated recently ${ }^{6,7}$ that the FiniteElement Method $(\mathrm{FEM})^{8}$ is a powerful and versatile tool to study magnetotransport in inhomogeneous samples of various shapes; here, we use the FEM analysis to model not only the geometric factor and $K_{H}$ in inhomogeneous Hall sensors but also the thermal drift of $K_{H}$. As the thermal drift is small (a few hundreds of ppm in the Hall signal), the calculation of the thermal drift is much more challenging than the simple calculation of $K_{H}$ at fixed $T$. However we find that the FEM gives an excellent agreement with the experimental results, which proves both i) the quality of the samples and ii) the usefulness of the predictive FEM method when designing devices.

\section{MODEL}

As the thermal drift mainly originates from the surface states, the idea of implementing a gated Hall device in the form of a pseudomorphic High Electron Mobility Transistor (pHEMT) looks attractive ${ }^{9}$, as the Fermi energy at the surface will be controlled by the gate voltage. Fig. 2a shows such a typical device, embedded in a circuit, where the gate covers the central part of the Hall cross. As the distance between the gate and the 2DEG channel is much smaller than all the considered distances in the plane of the cross, the problem will be considered as two-dimensional. At any point $(x, y)$ under the gate, the 2DEG concentration $n(x, y)$ is given by

$$
n(x, y)=\frac{C}{e}\left(V_{g}-V(x, y)\right)+n_{0}
$$

where $C$ is the capacitance per unit area, $n_{0}$ is the 2DEG concentration without gate introduced previously, $V_{g}$ is the gate voltage, $V(x, y)$ is the local voltage of the 2DEG. The capacitance $C$ is a planar capacitance given by the thickness and the permittivity of the heterostructure between the gate and the 2DEG. Such a sensor behaves like a transistor and in the linear regime, when the drain-to-source voltage $V_{\text {in }}$ is small: $V_{\text {in }} \ll V_{g}+e n_{0} / C$, the charge density is constant under the gate and the potential $V$ at the middle of the Greek 


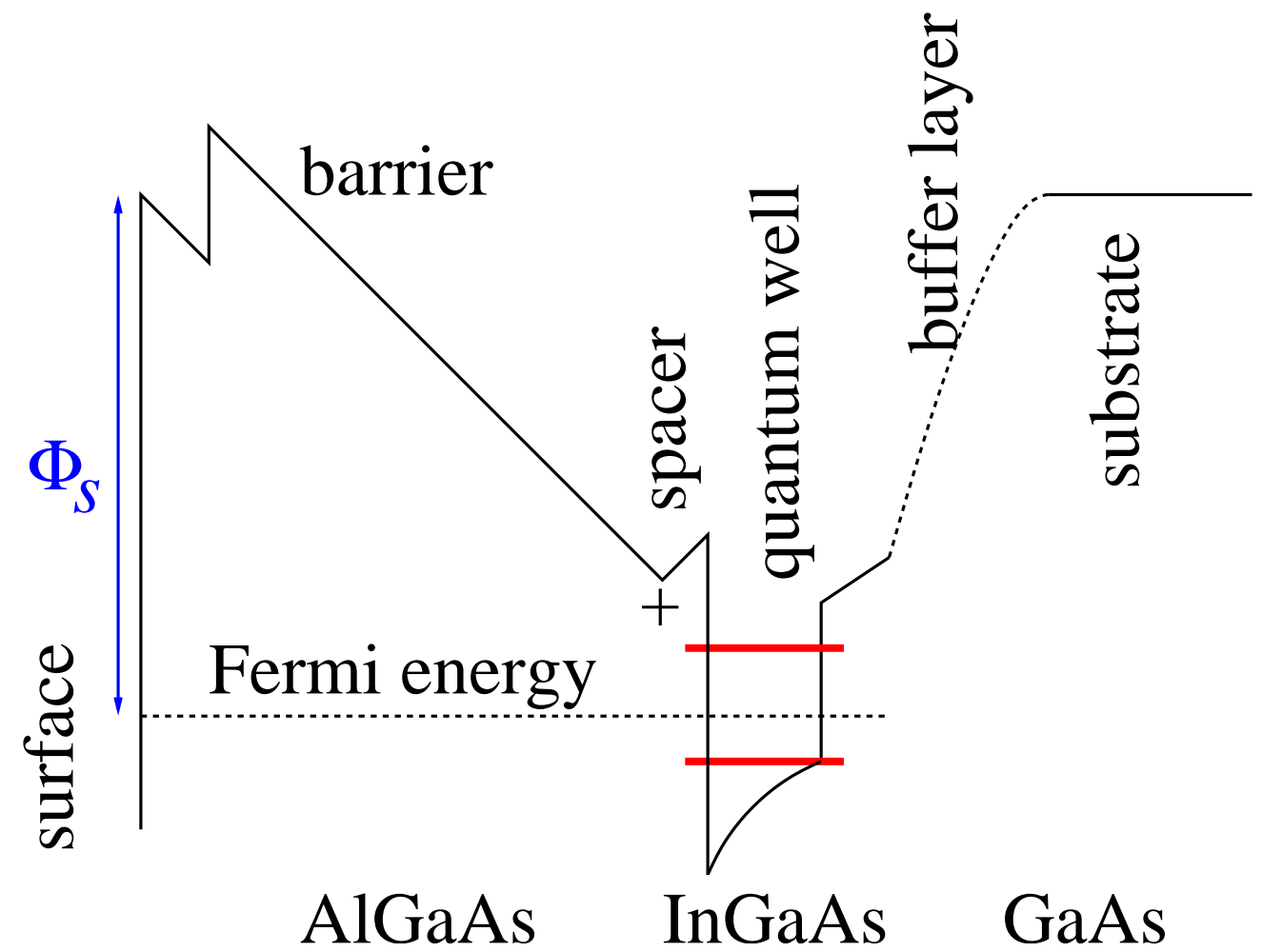

FIG. 1: (Color online) Sketch of the conduction band of a pseudomorphic AlGaAs/InGaAs/GaAs heterostructure with a delta-doping layer (+ sign).

cross can be approximated by

$$
V \approx R I / 2 \approx V_{i n} / 2
$$

where $R$ is the resistance of the sample at a given gate voltage. In a first approximation, the influence of the gate as well as the current in the lateral probes are neglected and the resistance is given by $\mathrm{R} \approx \rho L / W, L$ is the length of the cross, $W$ the width of the arm cross, $\rho$ the resistivity of the 2DEG. As the 2DEG concentration depends only weakly on $T$, the $T$-dependence of $\rho$ is the inverse of the $T$-dependence of the mobility, which is known to behave like $1 / T$ because of the lattice vibrations ${ }^{10}$.

The gate voltage $V_{g}$ can be controlled by different ways, one of them is indicated in Fig. 2a, where a resistor bridge with $R_{c} \gg R$ is used in order to have a gate bias proportional to the drain-to-source voltage $V_{i n}$ :

$$
V_{g}=\beta V_{i n},
$$

and the coefficient $\beta$ can be fixed at an arbitrary value by choosing the appropriate combination of resistors. A more complicated configuration, presented in Fig. 2b, will also be 
(a)
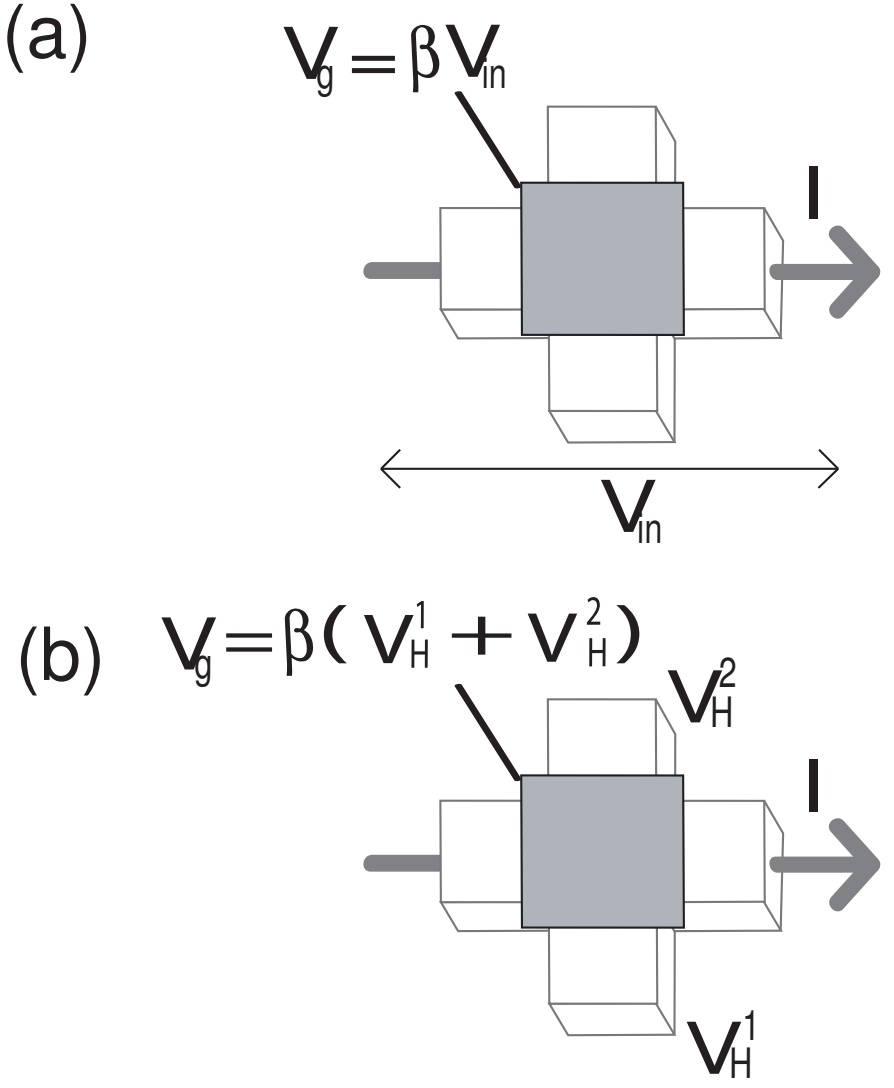

(c)

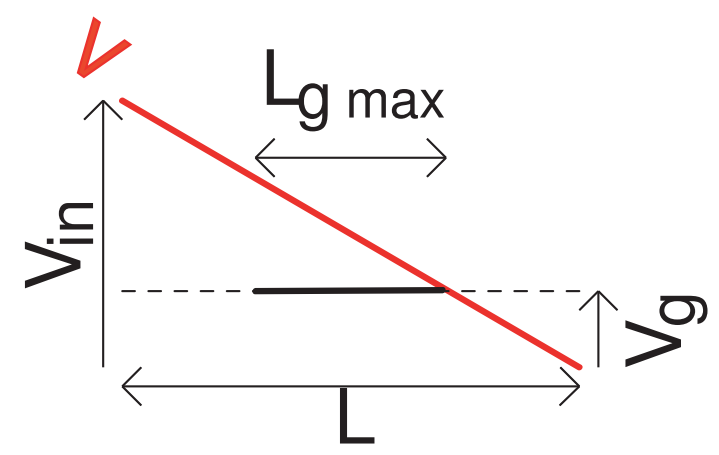

FIG. 2: (Color online) Sketches of the electrical connections. (a): the gate voltage is proportional to $V_{i n}$; (b) the gate voltage is proportional to $V_{H}^{1}+V_{H}^{2}$; (c) the red line represents the voltage drop in the Greek cross along the direction of the current flow.

considered. In Fig. 2b, the gate voltage is proportional to the common voltage of the Hall electrodes: $V_{g}=\beta V_{H}^{C}$, where $V_{H}^{C}=V_{H}^{1}+V_{H}^{2}$ and $V_{H}^{1}$ and $V_{H}^{2}$ are the voltages of the Hall probes. Note that in the linear regime, these two configurations are equivalent and lead to Eq. 4. We now include Eqs. 3 and 4 in Eq. 2 and we differentiate with respect to the 
temperature. We get:

$$
\frac{d n}{d T}=\frac{C}{e} \frac{d R}{d T}\left(\beta-\frac{1}{2}\right) I+\frac{d n_{0}}{d T} .
$$

Let us now assume that the Hall sensitivity is simply given by the concentration $n$ at the cross center: $K_{H}=1 /(n e)$. From Eq. 5, the thermal drift $S_{T}=-\left(1 / n_{0}\right) d n / d T$ equals zero at a critical current $I_{c}$ given by:

$$
I_{c}=n_{0} S_{T}^{0}\left(\frac{C}{e} \frac{d R}{d T}\left(\beta-\frac{1}{2}\right)\right)^{-1},
$$

where we defined the thermal drift $S_{T}^{0}$ as $S_{T}^{0}=-\left(1 / n_{0}\right) d n_{0} / d T$. The resistance $R$ and its thermal variation basically depends on the heterostructure while, by contrast, $\beta$ is easily modified by choosing another combination of resistors in Fig. 2. In order to maximize the magnetic gain $K_{H} I, I_{c}$ should be as high as possible so it can be necessary to reduce the value of $|\beta-1 / 2|$ for some applications. However, the introduction of the coefficient $\beta$ introduces also additional complications because the gate cannot cover the totality of the Greek cross anymore. Indeed, in this case the part of the cross which is close to the drain would have a gate voltage much higher than the underlying channel voltage, and the device would suffer important current leaks between the channel and the gate. Fig 2c illustrates the need for smaller gates by depicting a linear voltage drop in the 2DEG. This figure shows that the current leaks are negligible if $\beta<\left(L-L_{g}\right) / 2 L, L_{g}$ the width of the gate reduced to the central part of the cross. Besides, noise reduction and offset elimination require spinning current and contact rotations ${ }^{11}$, which implies that the Hall devices must keep their fourfold symmetry with respect to the cross center. Therefore the gate has to be removed symmetrically from the four arms of the cross.

We stress now that Eq. 5 is only a crude estimation of the critical current $I_{c}$. First of all, Eq. 5 is valid only in the linear regime of the device, at very low drain-to-source voltage $V_{i n}$. However, we try to obtain $I_{c}$ as high as possible, so this condition cannot be fulfilled. When the current increases, the potential bends under the gate and the channel voltage at the cross center does not equal $V_{i n} / 2$ anymore. Second, Eq. 5 is a one-dimensional approximation, which neglects the current in the lateral probes assumes a geometric factor $G=1$. These issues have no analytical solution; the proper way to overcome them is to develop a finite element analysis of the device. 


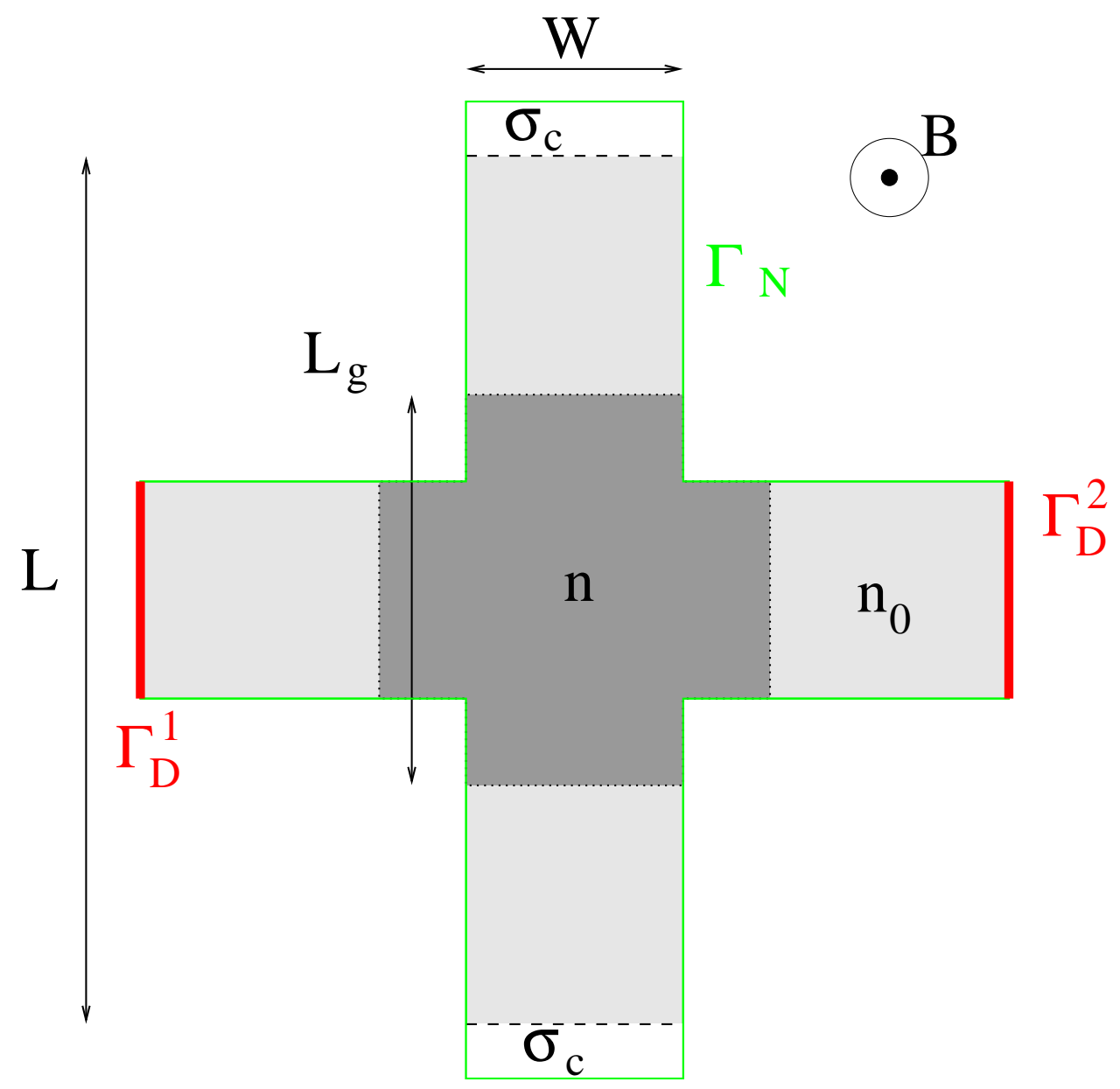

FIG. 3: (Color online) Sketch of a typical Hall cross. The domain is mainly divided into two parts: dark gray: 2DEG with gate (carrier density $n$ ), light gray: 2DEG without gate (carrier density $n_{0}$ ). A third part (white) defines the lateral probes and is used only to define the proper boundary conditions. The boundary conditions are also indicated: by red lines for Dirichlet conditions (voltage fixed) and by green lines for Neumann conditions (current parallel to the boundary).

\section{FINITE ELEMENT ANALYSIS}

The current flow in the structure is given by the Ohm's law

$$
\mathbf{j}=\sigma \mathbf{E},
$$

where $\mathbf{j}$ is the current density, $\mathbf{E}$ is the electric field, $\sigma$ is the conductivity matrix:

$$
\sigma=\frac{\sigma_{0}}{1+\mu^{2} B^{2}}\left(\begin{array}{cc}
1 & -\mu B \\
\mu B & 1
\end{array}\right),
$$




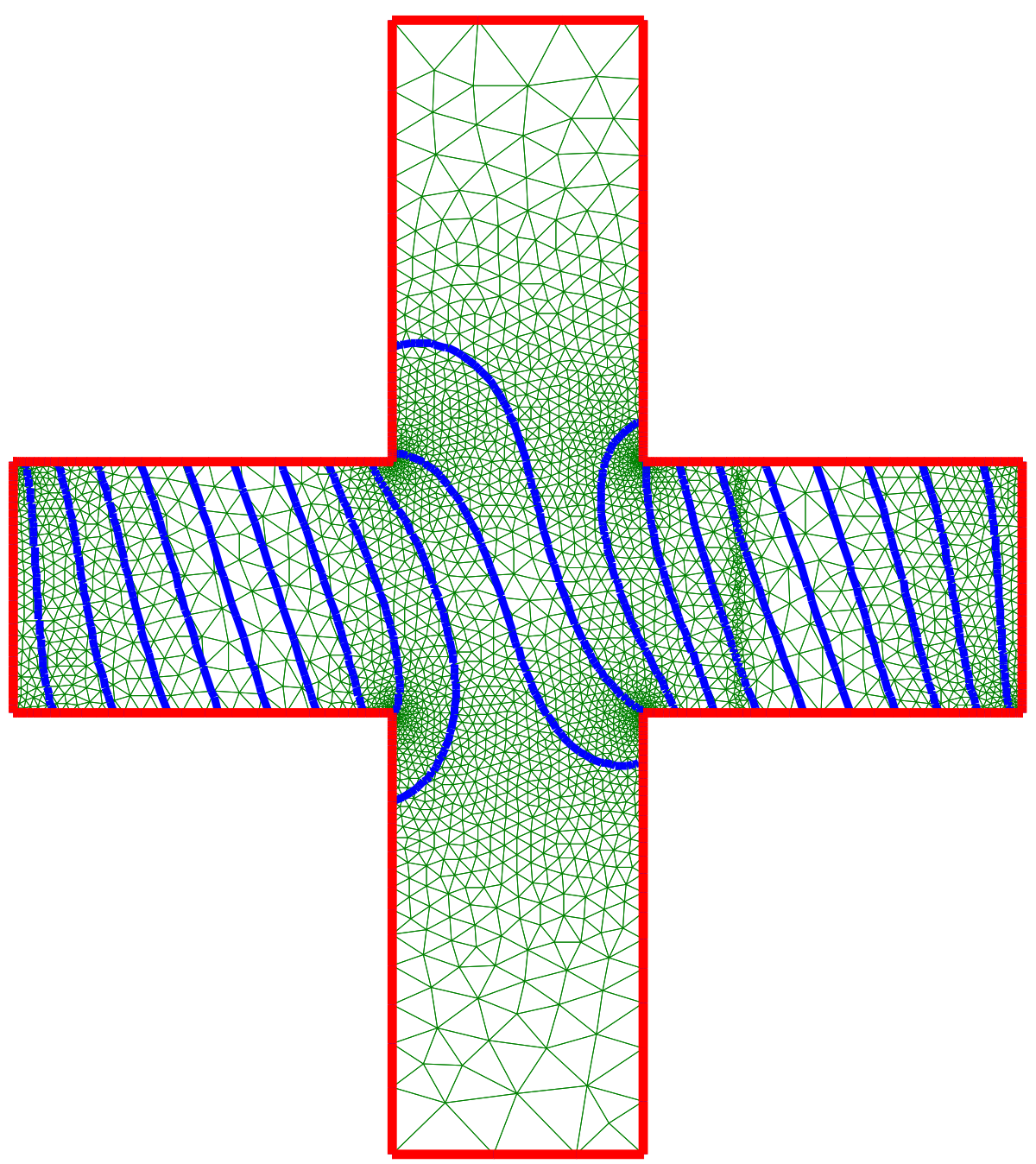

FIG. 4: (Color online) In-plane results of the FEM calculation for sample S3b with $L / W=$ 4, $B=0.3 \mathrm{~T}, V_{i n}=1 \mathrm{~V}, V_{g}=0 \mathrm{~V}$. Blue: domain boundaries, revealing the gate and the lateral probes. Black: triangular mesh. Green: contour lines for the potential with a step of $0.05 \mathrm{~V}$. Real calculations involved a finer mesh.

$\mu$ is the drift mobility, $B$ is the magnetic field applied perpendicular to the sample plane, $\sigma_{0}$ is the Drude conductivity at $B=0 \mathrm{~T}$ given by:

$$
\sigma_{0}=n e \mu
$$

and $n$ is the carrier density.

Combining the Ohm's law with the continuity equation $\nabla \cdot \mathbf{j}=0$, the steady state equation 
to be solved is

$$
\nabla \cdot \sigma \nabla V=0
$$

where $V$ is the electrical potential $\mathbf{E}=-\nabla V$. Eq. 10 is an elliptic equation whose solution is uniquely determined if adequate boundary conditions are fixed. The domain and the chosen boundary conditions are indicated in Fig. 3. The domain in which the solution is computed includes the Hall Greek cross with the two lateral Hall probes. At the current leads, the voltages are fixed, which gives rise to the Dirichlet boundary conditions indicated by the red lines in Fig. 3. On the other boundaries, indicated by green lines, we impose that no current flows across the boundaries. This corresponds to the Neumann boundary conditions. Note that these conditions are only valid for Hall measurement, when no current flows through the voltmeter used to measure the Hall voltage.

We detail how to express the conductivity in these inhomogeneous samples. Fig. 3 shows that the Greek cross is divided into two parts:

1. the arms of the Hall cross, which are not covered by the central gate, where the carrier density is given by $n=n_{0}$ and the conductivity by $\sigma=n_{0} e \mu$;

2. the central part of the cross, which is covered by the gate. Here the carrier density is given by Eq. 2 .

A third part defining the lateral ohmic contacts is used to simulate the boundary condition of the lateral probes: no net current going through and a constant voltage. These contacts are defined with an arbitrary conductivity $\sigma_{c} \gg \sigma_{0}$ independent on $B$.

Eqs. 2, 9 and 10 are coupled and define a non linear problem which can be solved by a relaxation method. Still, the current $I$ flowing through the structure has to be evaluated in order to compute the geometric factor $G$. Numerically it is simpler to evaluate the current flowing through one of the boundary corresponding to the current leads:

$$
I=\int_{\Gamma_{D}^{1}} \nabla V \cdot \mathbf{n} d l
$$

where $\mathbf{n}$ the normal to the boundary.

We used the software FreeFem++ to define the mesh and solve the problem. For illustration, Fig. 4 shows a typical mesh (green) generated for a structure with a $L / W$ ratio of 4 and a gate polarization $V_{g}=V_{i n} / 3$. The isolines of the calculated potential are also shown in 


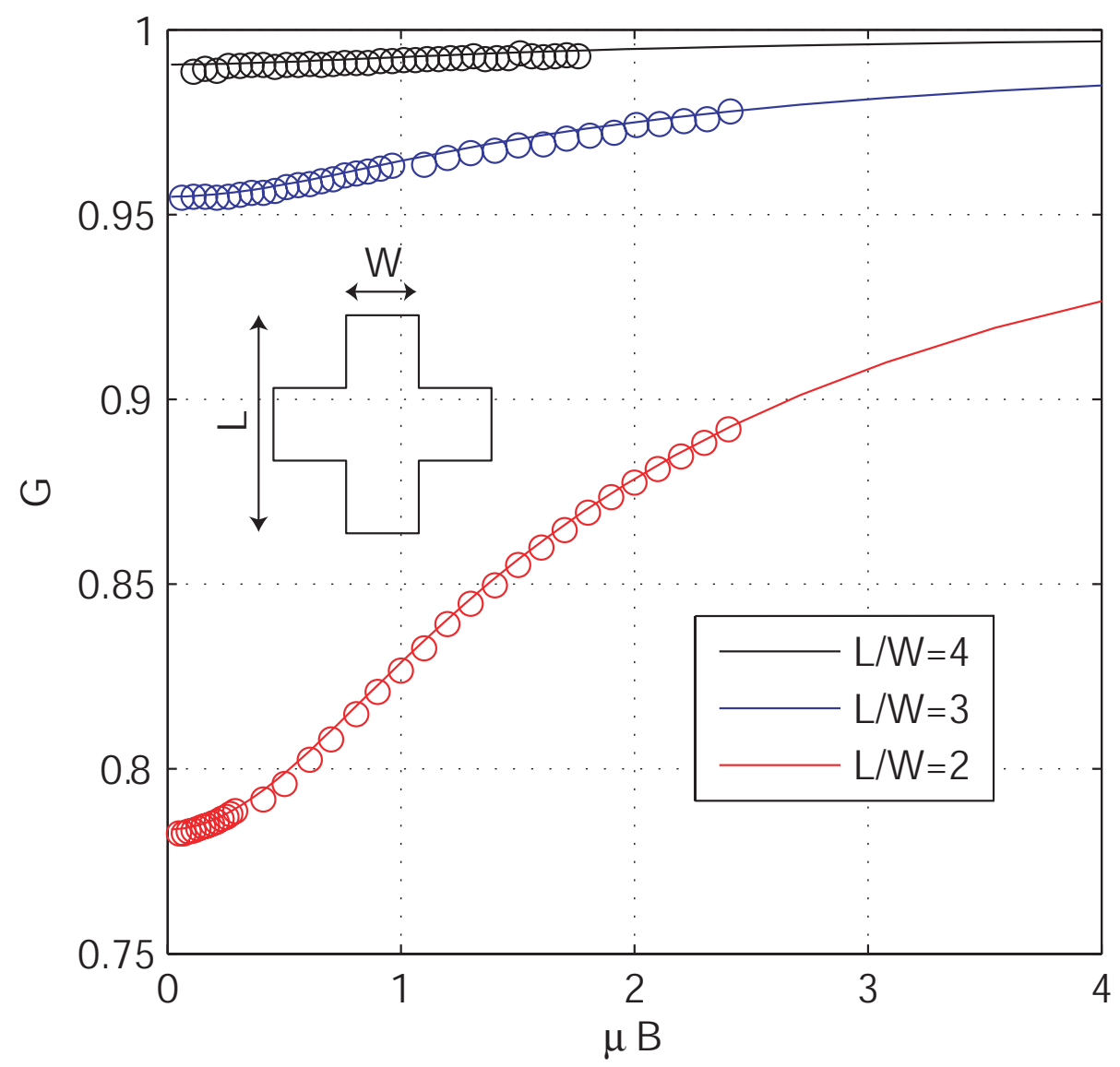

FIG. 5: (Color online) Comparison of the FEM method (open circles) with the analytic method (lines) for an homogeneous Greek cross with three different aspect ratio: $L / W=4$ (black), $L / W=$ 3 (blue), $L / W=2$ (red).

thick blue lines. The quality of the mesh is of prior importance to obtain a good estimation of the Hall coefficient. We employed an additional Delaunay automatic remeshing algorithm to minimize the errors and to refine the precision of the calculation.

We also compared the results of the calculation with well known analytical results, for simplified geometries. Fig. 5 shows the coefficient $G(\mu B)$ for 3 homogeneous Greek crosses without central gate and with 3 different aspect ratio: $L / W=2,3$ and 4 . The exact analytical solution have been calculated by $\operatorname{Versnel}^{12,13}$ and is indicated in Fig. 5 by solid lines. Our numerical results obtained by FEM are reported by open dots. The discrepancy between the two methods is less than $0.2 \%$. At higher fields $(\mu B>2)$ the FEM becomes time an memory consuming because of the appearance of singularities at the corners of the 

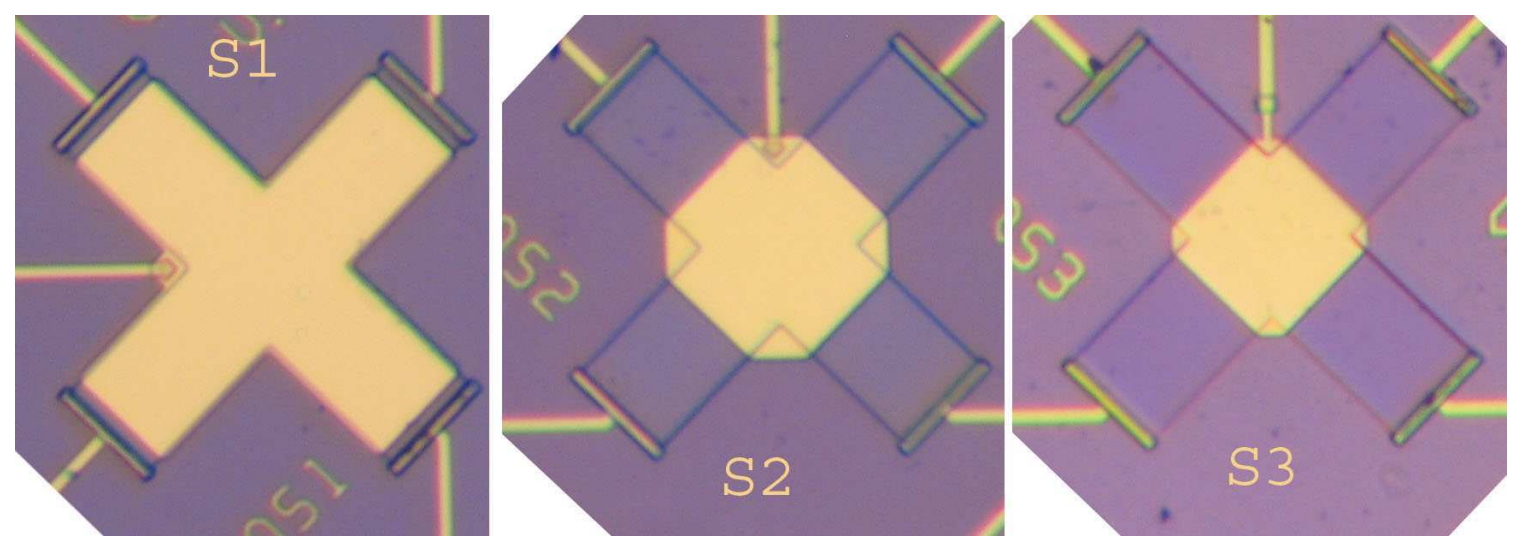

FIG. 6: (Color online) SEM photographes of the three samples with different gate geometry. Left panel: plain gate (geometry S1) ; middle panel: large central gate (S2); right panel: small central gate (S3).

\begin{tabular}{|l||cccc|c|}
\hline process & $n_{0}$ & $\begin{array}{c}\mu_{0} \\
\mathrm{~m}^{2} . \mathrm{V} \cdot \mathrm{s}^{-1}\end{array}$ & $\begin{array}{c}V_{T} \\
\mathrm{~V}\end{array}$ & $\begin{array}{c}S_{T}^{0} \\
\mathrm{ppm} / \mathrm{K}\end{array}$ & $\begin{array}{c}\frac{d R}{d T} \\
\Omega \cdot \mathrm{K}^{-1}\end{array}$ \\
\hline \hline $\mathrm{a}$ & 7.3 & 0.71 & -2.9 & -205 & 13 \\
$\mathrm{~b}$ & 7.3 & 0.71 & -2.7 & -187 & 15.6 \\
\hline
\end{tabular}

TABLE I: Measured parameters for samples of geometry S1 originating from different wafers. Parameters $\mathrm{n}_{0}, \mu_{0}$ and $\mathrm{V}_{T}$ are measured at $300 \mathrm{~K}, S_{T}^{0}$ is measured over the temperature range $\left[-55^{\circ} \mathrm{C}, 100^{\circ} \mathrm{C}\right]$ for process $a$ and over $\left[-55^{\circ} \mathrm{C}, 125^{\circ} \mathrm{C}\right]$ for process $b . \frac{d R}{d T}$ (only used in a refinement of the $C_{\text {eff }}$ method) is measured at $\mathrm{V}_{g}=0 \mathrm{~V}$.

drain and source electrodes. Results of Fig. 5 have been computed on a $2.4 \mathrm{GHz}$ computer, the number of nodal points was limited to 10000 and the calculation of a single value of $K_{H}$ takes approximately two minutes.

\section{COMPARISON WITH EXPERIMENTAL RESULTS}

\section{A. Sample description}

The structures used for this study are delta-doped AlGaAs/InGaAs/GaAs pseudomorphic heterostructures grown by Molecular Beam Epitaxy on GaAs wafers. The active layer is an 
$\mathrm{In}_{0.15} \mathrm{Ga}_{0.85} \mathrm{As}$ quantum well of width $20 \mathrm{~nm}$. The growth of the $\mathrm{QW}$ is followed by an $\mathrm{Al}_{x} \mathrm{Ga}_{1-x} \mathrm{As}$ cap layer, whose the $\mathrm{Al}$ concentration $x$ varies as a function of the depth in order to avoid the formation of parasitic channel. The growth is terminated by an additional GaAs layer. The total thickness of the cap layer and the GaAs layer is $240 \mathrm{~nm}$. Electrons in the QW are provided by a Si-doping layer, located $40 \mathrm{~nm}$ from the QW. A sketch of the band diagram is indicated in Fig. 1.

Most of the Greek crosses are realized by mesa etching (process a), some of them (process b) by ion implantation. Standard AuGeNi ohmic contacts are used. The gate is done from a Ti/Pt/Au layer. Overlying passivation includes a dielectric layer either of PECVD silicon dioxide or PECVD silicon nitride. Fig. 6 shows some of the samples analyzed in this study. The three Greek crosses of Fig. 6 have a form factor $L / W=4$ with $W=80 \mu \mathrm{m}$. For geometry S1 (Fig. 6 left), the gate covers the whole cross. For geometry S2 (Fig. 6 center), the gate width $L_{g}$ is equal to $140 \mu \mathrm{m}$; for geometry S3 (Fig. 6 right), $L_{g}=110 \mu \mathrm{m}$. Additional reference samples, without gate, are also processed.

Samples are measured under an air flux at controlled temperature. The temperature can be fixed between $-60^{\circ} \mathrm{C}$ and $150^{\circ} \mathrm{C}$ with a temperature precision of the order of $0.1 \mathrm{~K}$. The magnetic field is generated by a resistive solenoid. Most of the Hall measurements are taken at $B=0.106 \mathrm{~T}$.

A summary of the sample characteristics is given in table I for the two different processes. Note that the electron concentration $n_{0}$ and the corresponding drift $S_{T}^{0}$ can be measured by two methods: i) either by the use of an additional reference sample without gate, or ii) on the samples to be studied, at very low drain-to-source voltage and with $V_{g}=0 \mathrm{~V}$. Experimentally, we found that the two methods gave slightly different results, possibly because of the influence of the metal work function ${ }^{14}$. Therefore, in table I, only $n_{0}$ and $S_{T}^{0}$ obtained by the second method are reported. On average, we measured $n_{0} \approx 7.3 \times 10^{11} \mathrm{~cm}^{-2}$, $\mu \approx 0.71 \mathrm{~m}^{2} \cdot \mathrm{V}^{-1} \cdot \mathrm{s}^{-1}, S_{T}^{0} \approx-200 \mathrm{ppm} / \mathrm{K},(d R / d T) / R \approx 4000 \mathrm{ppm} / \mathrm{K}$ and a threshold voltage $V_{T}=-e n_{0} / C \approx-2.9 \mathrm{~V}$. From these values we calculated $C \approx 4 \times 10^{-4} \mathrm{~F} \cdot \mathrm{m}^{-2}$, in agreement with the theoretical value deduced from the heterostructure parameters. From Eq. 6 one therefore expects a critical current $I_{c} \approx 70 \mu \mathrm{A}$ for $\beta=0$ and $I_{c} \approx 200 \mu \mathrm{A}$ for $\beta=1 / 3$.

The two processes gave very similar results; in this paper, Figs. 7 and 8 correspond to several samples from process a; the other experimental figures correspond to an unique sample from the second process b. 


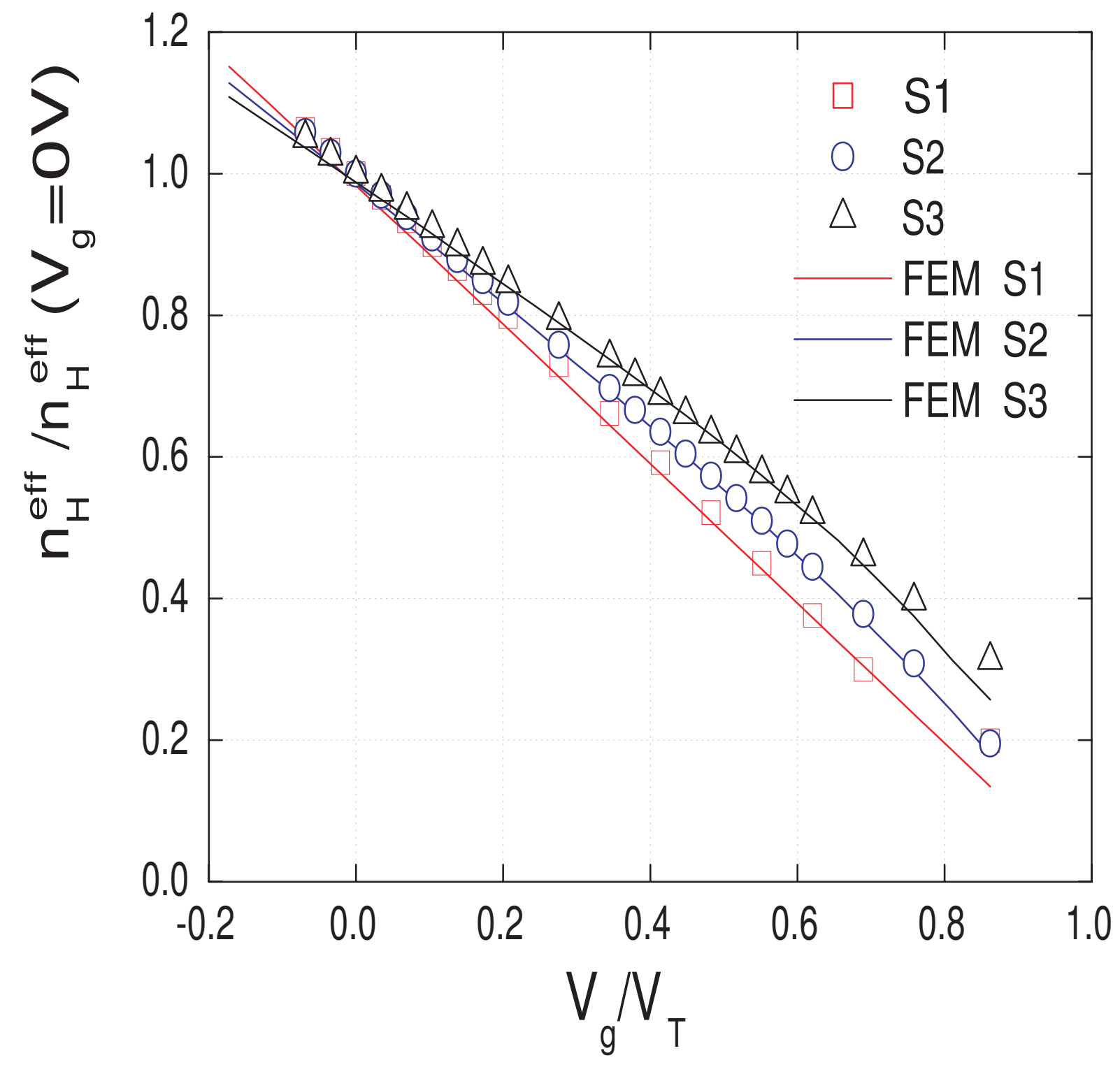

FIG. 7: (Color online) $n_{H}^{\text {eff }}\left(V_{g}\right)$ measured for the three different geometries S1,S2 and S3. Open symbols: experimental data, lines: FEM results.

\section{B. Data vs Experiment at fixed $T$}

Fig. 7 shows the normalized Hall density $n_{H}^{\mathrm{eff}}=1 /\left(K_{H} e\right)$ measured at room temperature as a function of the normalized gate voltage $V_{g} / V_{T}$ for a vanishing drain-to-source voltage: $V_{i n} \ll V_{T}$. The Hall density has been normalized with respect to its value at $V_{g}=0$. For most of the gate voltages presented in Fig. 7, the gated Hall sensors are in the linear regime. However, the three geometries S1, S2 and S3 give systematically different results and their 


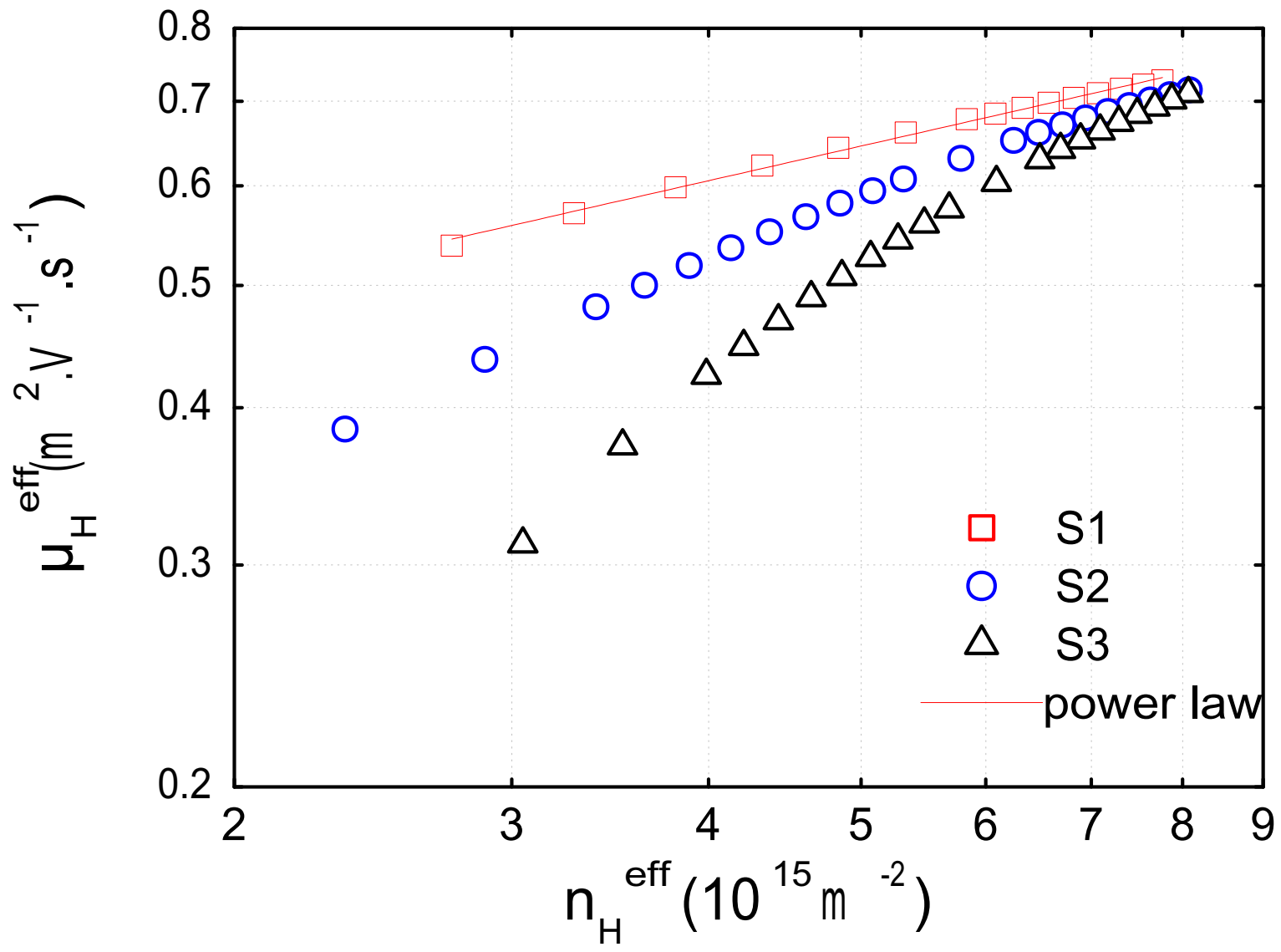

FIG. 8: (Color online) Open symbols: $\mu_{H}^{\mathrm{eff}}\left(n_{H}^{\mathrm{eff}}\right)(\neq \mu(n))$ measured for samples with geometries S1, S2 and S3. Solid line: power law fit for geometry S1.

$n_{H}^{\text {eff }}\left(V_{g}\right)$ do not superimpose. The explanation is as follows: only samples with geometry $\mathrm{S} 1$, where the $2 \mathrm{DEG}$ is completely covered by the gate, are homogeneous and have a geometrical correction $G \approx 1$ (see Fig. 5 with $\mathrm{L} / \mathrm{W}=4$ near the origin). For the two other geometries S2 and S3, measuring $n_{H}^{\text {eff }}$ without taking into account this correction $G$ leads to a dramatic error in the real 2DEG concentration. Fig. 7 also shows the result of the FEM calculations for the three geometries as solid lines. The calculations are obviously in excellent agreement with the data. We also stress that the calculation involves no adjustment parameter, as the vertical axis is normalized and that the horizontal axis may be expressed as a function of $V_{T}\left(1-\sigma_{g} / \sigma_{0}\right)$, where $\sigma_{g}$ is the conductance of the 2DEG under the gate, and the threshold voltage $V_{T}$ is measured independently, by linear extrapolation of the data obtained for the geometry $S 1^{15}$. The results of Fig. 7 have been reproduced for the two processes.

For the following comparisons, great care was taken to extract the mobility $\mu$ as a function 